Received: 2015.03 .17 Accepted: 2015.04.06 Published: 2015.09 .10
Authors' Contribution: Study Design A Data Collection B Statistical Analysis C Data Interpretation D Manuscript Preparation E Literature Search F Funds Collection G

\title{
Preoperative CT-Angiography Predicts Ex Vivo Vein Length for Right Kidneys After Laparoscopic Donor Nephrectomy
}

ABCDEF 1 Denise M.D. Özdemir-van Brunschot

$A B E 1$ Simone J. Rottier

ABE 1 Judith E. den Ouden

ABEF 1 Michel F. van der Jagt

ABEF 2 Frank C. d'Ancona

BE 3 Heinrich Kloke

AEF 1 Daan J.A. van der Vliet

BEF 4 Leo J. Schultze Kool

ABCDEF 1 Michiel C. Warlé
1 Department of Surgery, Radboud University Medical Center, Nijmegen, The Netherlands

2 Department of Urology, Radboud University Medical Center, Nijmegen, The Netherlands

3 Department of Nephrology, Radboud University Medical Center, Nijmegen, The Netherlands

4 Department of Radiology, Radboud University Medical Center, Nijmegen, The Netherlands
Corresponding Author: Source of support:
Denise M.D. Özdemir-van Brunschot, e-mail: denise.ozdemir-vanbrunschot@radboudumc.nl

Departmental sources

Background: Implantation of a kidney with a short renal vein is technically more challenging and therefore prone for technique-related complications. It remains unclear whether pre-operative computed tomography angiography (CTA), to assess vascular anatomy of the donor kidney, can be used to predict renal vein length.

Material/Methods: Right and left renal vein lengths of 100 consecutive kidney donors were measured in an oblique-coronal plane multiplanar reconstruction image of 100 consecutive kidney donors in whom ex vivo vein length was measured after recovery. In a second retrospective cohort of 100 consecutive kidney donors donating a right kidney, preoperative CTA vein length measurements were correlated to anastomosis time and early graft outcome.

Results: Left and right renal vein lengths, measured on CTA, were $43.2 \mathrm{~mm}$ and $30.0 \mathrm{~mm}$, respectively. No correlation was found between CTA and ex vivo measurements for the left renal vein ( $p=.610)$, whereas a significant correlation was found for the right renal vein $(p=.021)$. In the retrospective cohort, right renal vein length was significantly correlated with the anastomosis time but not with early graft outcome.

Conclusions: The length of the right, but not the left, renal vein can be predicted by preoperative CTA, but this does not hold true for the left renal vein.

MeSH Keywords: Kidney Transplantation • Living Donors • Renal Veins • Tomography Scanners, X-Ray Computed

Abbreviations: WIT2 - second warm ischemia time; CRR2 - creatinine reduction rate day 1 to day 2; CTA - 2-dimensional computed tomography angiography; DGF - delayed graft function; SGF - slow graft function; MPR - multiplanar reconstruction

Full-text PDF: http://www.annalsoftransplantation.com/abstract/index/idArt/894131

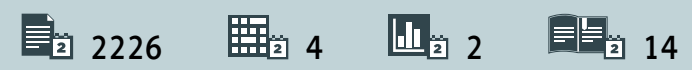




\section{Background}

The number of living donor kidney transplantations is increasing and living donor kidneys have become indispensable to overcome the organ shortage. In general, organ quality of living donor kidneys is superior when compared to kidneys from deceased donors. This is reflected by a longer graft and patient survival [1]. In order to obtain as much kidneys as possible, many transplant centers are extending their criteria for live kidney donation, e.g. with regard to donor age and complex arterial and venous anatomy. With regard to renal vein anatomy, most centers preferentially perform left sided donor nephrectomy, since left kidneys have longer veins than their right counterparts. The relatively shorter right renal veins may complicate the venous anastomosis during implantation.

However, the left renal vein has more side branches, e.g. adrenal, lumbar and gonadal veins, which require more time for dissection and clipping during laparoscopic donor nephrectomy. In cases where the left kidney is not available for donation e.g. complex left arterial anatomy or marginal estimated remaining kidney function, the right kidney will be considered. The short and long term results after transplantation of the right and left renal graft is similar [2-5].

Therefore, the choice for the left or right donor kidney, in case both kidneys are available from the donors' point of view, is based on centers' or surgeons' preference. However, the general opinion is that implantation of a kidney with a very short renal vein is technically more challenging and therefore prone for technique related complications. Especially in recipients with obesity it might be better to avoid transplantation of right kidneys with short renal veins.

Many centers perform computed tomography angiography (CTA) scans in all potential living kidney donors, to assess vascular anatomy; however the two-dimensional CTA measurement of renal vein length does not necessarily correlate with ex vivo renal vein length after kidney extraction and back table preparation. Therefore, the first research question is whether CTA measurement of renal vein length prior to laparoscopic donor nephrectomy correlates with ex vivo renal vein length measurement after kidney extraction and back table preparation. To address this question, a prospective cohort of 100 consecutive living donor kidney transplantations will be analyzed. If CTA measurements indeed correlate with ex vivo measurements, the second question that arises is whether renal vein lengths, as measured by preoperative CTA, correlates with early kidney allograft outcome (e.g. delayed graft function, slow graft function and graft loss due to technical failure). To address this question a retrospective cohort of 100 consecutive living kidney donors, donating a right kidney, at our center were assessed.

\section{Material and Methods}

\section{Patients}

All potential live kidney donors were evaluated by a nephrologist, urologist and vascular surgeon. During a multidisciplinary discussion the pre-operative CTA of the donor was used for decision making with regard the donors' eligibility for living kidney donation. With regard to the side of donor nephrectomy, preoperative CTA also plays a pivotal role and provides information regarding arterial and venous anatomy, presence of renal cysts (or other parenchymal lesions) and estimated remaining renal function (kidney size). The first cohort consisted of 100 consecutive adult kidney donors operated on between May 2011 and November 2012, in who ex vivo measurements of the renal vein were performed. These measurements were correlated to pre-operative CTA measurements.

A second cohort was assessed, containing all patients who underwent a right laparoscopic donor nephrectomy performed in the period between January 2003 and August $2014(n=100)$. Patients were divided into three equal groups based upon the length of the right renal vein measured on CTA $(\leq 22.3$; $22.3-31.8 ; \geq 31.8 \mathrm{~mm}$ ) and correlated to recipient outcome parameters.

\section{Computed tomography angiography}

The Siemens SOMATOM Sensation 64 was used for all CTA measurements. The Siemens SOMATOM Sensation 64 CT scanner is a 64-slice scanner, with a slice width of $1.0 \mathrm{~mm}$ and an effective mAs of 105 . Contrast was given after a delay of 10-15 seconds at a flow rate of 3.0-3.5 mL/sec. Multiplanar MPR reconstructions (MPRs) in an oblique-coronal plane were made on a TeraRecon iNtuiton workstation (TeraRecon Inc., Foster City, USA).

\section{CTA measurements of the renal vein length}

Oblique-coronal plane MPR images were used for renal vein length measurements. Two independent researchers (SR and JO) performed 3 measurements for each donor:

- Length of right renal vein: a straight line between both poles of the right kidney (line A) and a straight line at the lateral border of the inferior vena cava (line B) were drawn. The length of the right renal vein was defined as the centre-lumen distance between line $A$ and $B$ (Figure 1A).

- Length of the left renal vein: a straight line between both poles of the left kidney (line C) and a straight line at the medial side of the inferior vena cava (line $D$ ) were drawn. The length of the left renal vein was defined as the centrelumen distance between line C and D (Figure 1B). 

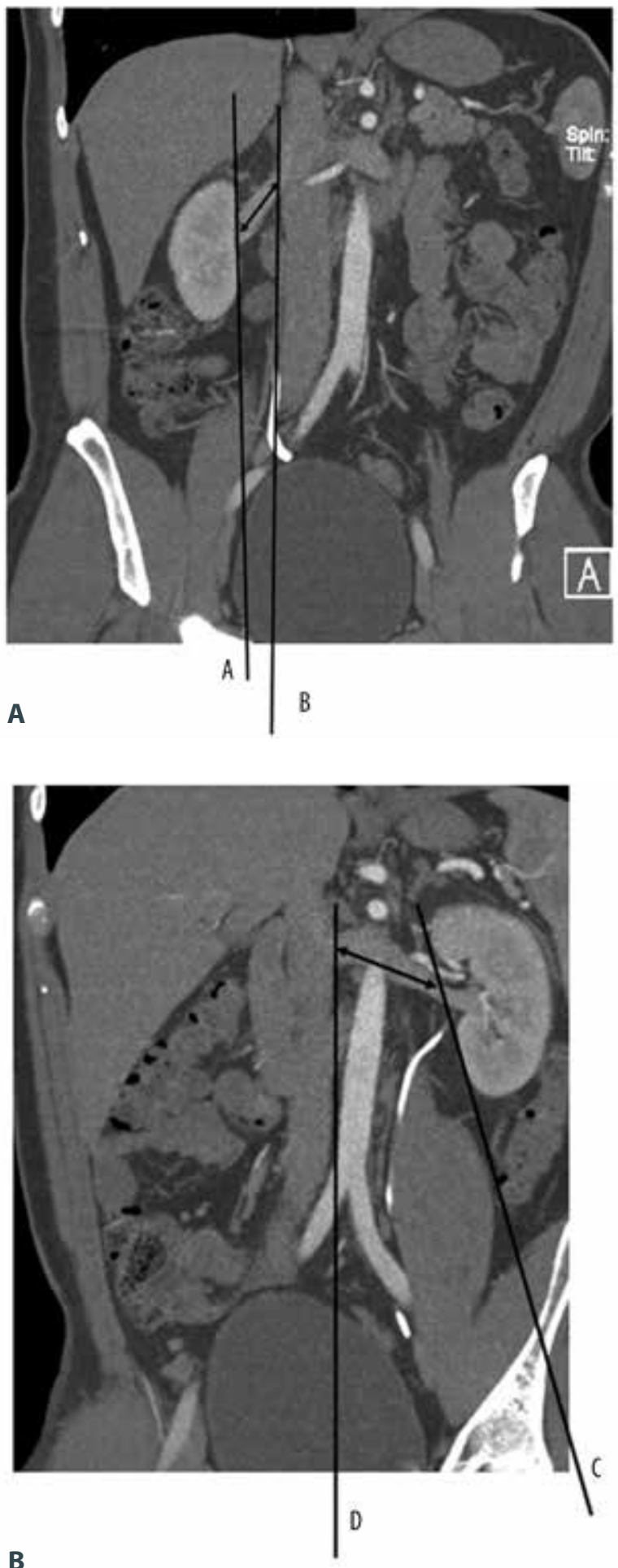

- Length of the left renal vein to the lateral border of the aorta: a straight line between both poles of the left kidney (line E) and a straight line at the lateral border of the aorta (line F) were drawn. During donor nephrectomy line F represents the transection zone of the left renal vein (Figure 1C).

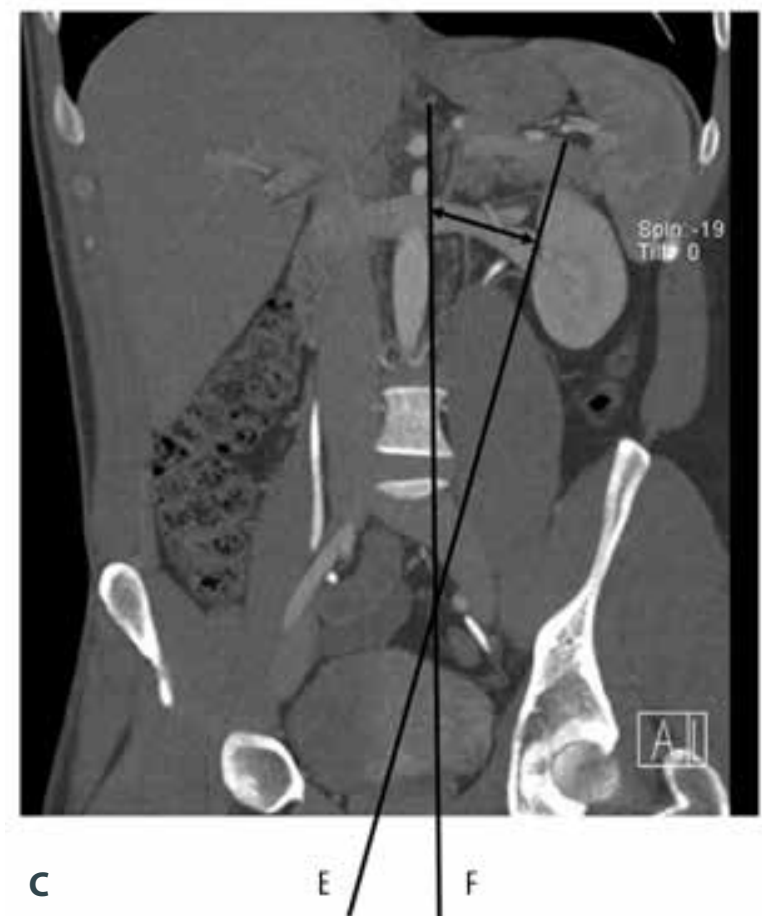

Figure 1. (A) Measurement of right renal vein on CTA in an oblique-coronal plane MPR image. (B) Measurement of left renal vein (from vena cava) on CTA in an obliquecoronal plane MPR image. (C) Measurement of left renal vein (from aorta) on CTA in an oblique-coronal plane MPR image.

In case of 2 renal veins, both veins were measured as described above.

\section{Surgical technique and ex vivo measurements}

Since 2003 the procedure of choice in our centre is transperitoneal laparoscopic donor nephrectomy without hand-assistance. First, a Hasson trocar is introduced and pneumoperitoneum is established. Subsequently, a $30^{\circ}$ video endoscope and 3 or 4 additional trocars are introduced. The hepatic or splenic flexure of the colon is mobilized, using ultracision. Gerota's fascia is opened and the renal vein, artery, and ureter are identified and dissected. The renal vessels are transected using an TA-endostapler. After recovery of the donor kidney, the donor kidney is flushed with cold perfusion fluid. Subsequently, redundant tissue surrounding the renal artery and vein is removed and small venous side branches are ligated. Thereafter, the ex vivo renal artery and vein lengths are measured under slight traction by 2 surgeons (MJ or MW). All procedures were performed by a surgical team consisting of a fixed combination of 2 urologists (Fd'A and $\mathrm{HL}$ ) and 2 vascular surgeons (MvdJ and MW) [6]. Regarding the implantation of the renal allografts, the vascular part of all procedures was performed by 2 vascular surgeons ( $M v d J$ and $M W$ ). 
Table 1. Demographics of donors and recipients; consecutive, prospective cohort $(n=100)$.

\begin{tabular}{|c|c|c|c|}
\hline & & Mean (SD) & Range \\
\hline \multirow{3}{*}{ Donor } & Age (years) & $52.5 \quad(11.4)$ & $25-76$ \\
\hline & Male/female & $48 / 52$ & \\
\hline & Body mass index & $25.5 \quad(3.1)$ & $18.0-34.5$ \\
\hline \multirow{3}{*}{ Recipient } & Age (years) & $45.6 \quad(15.6)$ & $6-73$ \\
\hline & Male/female & $53 / 47$ & \\
\hline & Body mass index & $24.3 \quad(4.1)$ & $15.3-35.2$ \\
\hline Side of nephrectomy & Left/right & $86 / 14$ & \\
\hline
\end{tabular}

Data are presented as number or mean $\pm \mathrm{SD}$ (standard deviation) and range or number.

Table 2. CTA and ex vivo renal vein measurements; consecutive, prospective cohort $(n=100)$.

\begin{tabular}{|c|c|c|c|c|c|c|c|c|c|}
\hline \multirow[b]{2}{*}{ Left renal vein (total) } & \multicolumn{2}{|c|}{$\begin{array}{l}\text { CTA in recovered } \\
\text { kidneys }\end{array}$} & \multicolumn{2}{|c|}{ Ex vivo } & n & $\begin{array}{c}\text { Difference CTA \& } \\
\text { ex vivo } \\
\text { measurements }\end{array}$ & \multicolumn{2}{|c|}{ CTA in all kidneys } & \multirow{2}{*}{100} \\
\hline & 69.8 & $(48-104)$ & & - & 86 & - & 70.2 & $(48-104)$ & \\
\hline Left renal vein (aorta) & 43.0 & $(22-70)$ & 30.7 & $(19-55)$ & 86 & $12.6(-33.8-9.8)$ & 43.2 & $(22-70)$ & 100 \\
\hline Right renal vein & 38.3 & $(27-57)$ & 26.3 & $(18-43)$ & 14 & $11.7 \quad(-.9-24.1)$ & 30.0 & $(9-57)$ & 100 \\
\hline
\end{tabular}

CTA - computed tomography angiography. All measurements expressed as $\mathrm{mm}$, data expressed as mean (range).

\section{Recipient demographics and outcomes}

Recipient outcome measures include: creatinine reduction ratio (CRR2), post-operative creatinine at day 5 , slow graft function (SGF), delayed graft function (DGF), graft loss due to technical failure. Technical failure was defined as transplantectomy within 10 days, without signs of perfusion on ultrasound and without evidence of rejection. DGF is the need for dialysis in the first week after surgery [7], SGF as serum creatinine $>3.0$ $\mathrm{mg} / \mathrm{dl}$ at day 5 without the need for dialysis [8].

CRR2 from day 1 to 2 (ccr2) is predicator for long-term graft outcomes and was calculated according to the following formula [9]:

$$
\operatorname{CRR} 2(\%)=\frac{(\text { creatinine day } 1-\text { creatinine day } 2) \times 100}{\text { creatinine day } 1}
$$

\section{Statistical analyses}

All statistical tests were performed with SPSS version 20.0 (SPSS Inc. Chicago, IL, USA). Continuous variables were expressed as mean $\pm S D$, numerical data was expressed as number and percentage. Numerical data were compared with Kruskall-Wallis test, categorical data with Fisher exact test. Correlations between CTA and ex vivo measurements was determined by
Spearman's correlation analysis. A p-value of 0.05 or less was considered to be significant.

\section{Results}

\section{CTA and ex vivo renal vein length measurements}

Baseline characteristics of the first, prospective cohort are shown in Table 1. Reasons to select the right instead of the left kidney for recovery were: multiple arterial anatomy (9 cases) or multiple venous anatomy (1 case) on the left side; renal cysts in the right kidney ( 2 cases) and small kidney stones in the right kidney (2 cases).

The CTA and ex vivo measurements are shown in Table 2. The length of the left renal vein measured on CTA was significantly longer when compared to the right renal vein, respectively $43.2 \mathrm{~mm}$ (range, 22 to 70 ) versus 30.0 (range, 9 to 57) (p<.05). Also, length of the left renal vein when measured ex vivo was longer, $30.7 \mathrm{~mm}$ versus $26.3 \mathrm{~mm}(\mathrm{p}=.05)$.

The mean difference between CTA and ex vivo measurements was $12.6 \mathrm{~mm}$ (range, -33.8 to 9.8 ) for left renal veins and $11.7 \mathrm{~mm}$ (range, -.9 to 24.1 ) for right renal veins. Spearman 


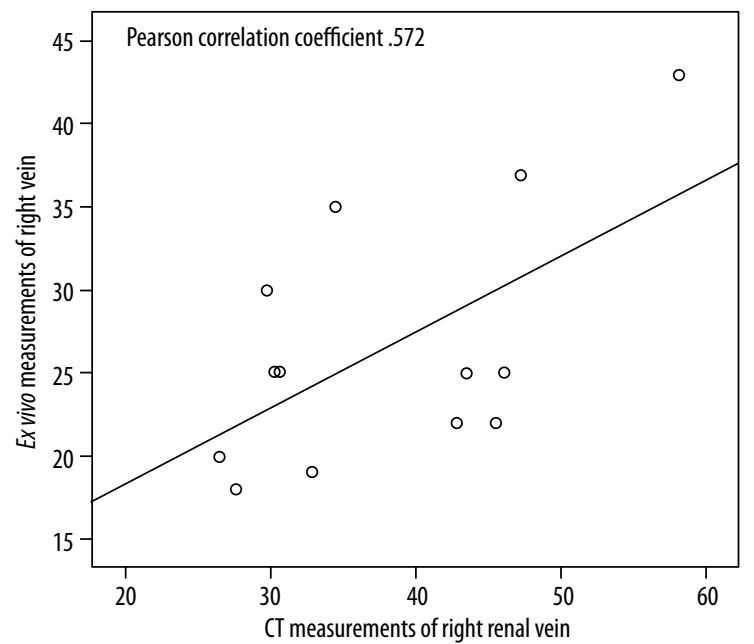

Figure 2. Ex vivo measurements and CT measurements of the right renal vein.

correlation coefficients ( $\rho$ ) between CTA and ex vivo measurements were.058 $(p=.610)$ for left renal veins and.572 $(p=0.041)$ for right veins (Figure 2).

\section{Right renal vein and recipient outcome}

Donor and recipient characteristics of the second, retrospective cohort are shown in Table 3 . The right renal veins were divided in 3 equal groups ( $\langle 22.3 ; 22.3$ to 31.8 , and $>31.8 \mathrm{~mm}$ ) and correlated to recipient outcomes (Table 4). In 100 patients, we found 6 technical failures. Of these 6 technical failures, 4 technical failures were present in the group with a length of 22.3 to $31.8 \mathrm{~mm}$. A significant shorter anastomosis time was seen in the group with the long renal veins $(p=0.021)$. Shorter right renal veins were not associated with graft loss due to technical failure.

\section{Discussion}

We believe this is the first study exploring the clinical value of renal vein length measurements on CTA. In a consecutive, prospective cohort, the length of the right renal vein based on CTA was significantly correlated with ex vivo measurements after back Table preparation, while the length of the left renal vein was not. The main explanation for this discrepancy is that the right renal vein is almost without exception transected, using the endostapler, at the level just lateral of the caval vein, whereas the level of transsection of the left renal vein is much more variable. In some cases the left renal vein is transected at the distal side of the adrenal vein, whereas in other cases it is transected much more proximal, e.g. 1 to $2 \mathrm{~cm}$ medial to the lateral border of the juxtarenal aorta.

The mean difference between CTA and ex vivo measurements was $12.6 \mathrm{~mm}$ (range, -48.6 to 16.4 ) for left and $11.7 \mathrm{~mm}$ (range, -.9 to 24.1) for right renal veins. This difference can be explained by the loss of length caused by the use of the TA stapling device. Jellison et al. have reported a loss of $10.0 \mathrm{~mm}$ when using an endo-TA stapler [10]. The right and left renal vein measured on CTA have a mean difference of $14.7 \mathrm{~mm}$, ex vivo the right and left renal vein differ by $4.1 \mathrm{~mm}$. This can be explained by the fact that right kidneys with very short renal veins (i.e. $15 \mathrm{~mm}$ ) are not accepted for donor nephrectomy in our center.

In many centers, including ours, preferably the left kidney is recovered. The left kidney is preferred since in general right kidneys have a shorter renal veins. The general opinion among transplant surgeons is that an implantation of kidneys with short renal veins is technically more challenging and may be associated with vascular complications. To overcome these technical difficulties associated with shorter renal veins, surgical modifications have been proposed, for example the use of Hem-o-locks instead of Endo-GIA for vein dissection and the use of hand-assistance for lateral traction [11].

Table 3. Demographics of donors and recipients and perioperative data, all right donor nephrectomies since 2003; retrospective cohort $(n=100)$.

\begin{tabular}{|c|c|c|c|c|}
\hline & & \multicolumn{2}{|c|}{ Mean (SD) } & Range \\
\hline \multirow{3}{*}{ Donor } & Age (years) & 53.1 & (11.4) & $19.8-72.9$ \\
\hline & Male/female & \multicolumn{2}{|c|}{$48 / 52$} & \\
\hline & Body mass index & 25.3 & (3.3) & $17.0-31.9$ \\
\hline \multirow{3}{*}{ Recipient } & Age (years) & 42.1 & (17.0) & $4.1-73.7$ \\
\hline & Male/female & \multicolumn{2}{|c|}{$54 / 46$} & \\
\hline & Body mass index & \multicolumn{2}{|c|}{23.5} & $14.3-44.0$ \\
\hline
\end{tabular}

Data are presented as mean \pm standard deviation and range or number. 
Table 4. CTA renal length of right renal allografts and recipient outcomes; retrospective cohort $(n=100)$.

\begin{tabular}{|c|c|c|c|c|c|}
\hline & & $\begin{array}{l}\leq 22.3 \\
(n=33)\end{array}$ & $\begin{array}{c}22.3-31.8 \\
(n=33)\end{array}$ & $\begin{array}{l}\geq 31.8 \\
(n=34)\end{array}$ & $\begin{array}{c}\text { P-value } \\
\text { (kruskal wallis or } \\
\text { fisher exact) }\end{array}$ \\
\hline \multirow{3}{*}{ Donor } & Age (years) & $48.1 \quad(11.0)$ & $51.8(13.0)$ & $54.2 \quad(9.6)$ & .077 \\
\hline & Male/female & $16 / 17$ & $15 / 18$ & $17 / 17$ & .931 \\
\hline & Body mass index & $25.4 \quad(3.3)$ & $25.8 \quad(2.7)$ & $24.6 \quad(3.7)$ & .275 \\
\hline \multirow{3}{*}{ Recipient } & Age (years) & $41.7(17.3)$ & $42.5(17.6)$ & $42.1(16.7)$ & .990 \\
\hline & Male/female & $19 / 14$ & $17 / 16$ & $18 / 16$ & .875 \\
\hline & Body mass index & $23.0 \quad(3.8)$ & $23.1 \quad(4.5)$ & $24.2 \quad(5.3)$ & .557 \\
\hline \multirow{7}{*}{$\begin{array}{l}\text { Outcome } \\
\text { parameters }\end{array}$} & WIT2 (minutes) & $28.6 \quad(9.2)$ & $24.2 \quad(9.1)$ & $23.7 \quad(8.6)$ & .021 \\
\hline & CRR2 (\%) & $36.0 \quad(20.1)$ & $39.5 \quad(21.2)$ & $38.2(19.2)$ & .522 \\
\hline & $\begin{array}{l}\text { Creatinine day } 5 \\
\text { (micromol/l) }\end{array}$ & $197 \quad(183)$ & $199 \quad(228)$ & $164 \quad(185)$ & .183 \\
\hline & $\begin{array}{l}\text { Creatinine year } 1 \\
\text { (micromol/ll }\end{array}$ & $114 \quad(30)$ & $117 \quad(28)$ & $144 \quad(150)$ & .888 \\
\hline & $\begin{array}{l}\text { Delayed graft } \\
\text { function }\end{array}$ & 0 & 2 & 1 & .353 \\
\hline & Slow graft function & 0 & 1 & 2 & .369 \\
\hline & Technical failure & 1 & 4 & 1 & .195 \\
\hline
\end{tabular}

Data represented as mean (standard deviation) or number. CTA - computed tomography angiography; WIT2 - second warm ischemia time; CRR - creatinine reduction.

In literature, no obvious differences in graft survival were found when comparing the implantation of right or left renal allografts $[3,4,11,12]$. However, some authors have described an association of the right kidney and prolonged second warm ischemia time $[4,13,14]$. This illustrates that the implantation of right donor kidneys is technically more demanding. Some transplant centers preferably recover right kidneys. The main reason for this is that during right laparoscopic donor nephrectomy dissection of side branches is seldom necessary [4] and therefore right kidney are easier to procure than left kidneys.

In our second, retrospective cohort the length of right renal veins, measured on CTA, was compared to recipient outcome measures. A significant longer WIT2 was observed for the group with the short renal veins, implying that the implantation of right allografts with shorter veins is technically more demanding. We observed no significant difference in recipient outcome parameters.

\section{Conclusions}

Important strengths of this study with regard to the primary research question, are related to its design as a consecutive, prospective cohort study. Ex vivo measurements of all the renal veins were performed by two surgeons (MW and MvdJ). Furthermore, to our knowledge, no other studies have been conducted exploring the clinical value of CTA vein measurements prior to laparoscopic donor nephrectomy. The limitation of our study is related to the retrospective nature of the second cohort. This may have introduced a certain degree of selection bias, as kidneys with short renal veins may have been avoided during the preoperative work-up. However, one would expect that the negative impact of short renal vein lengths would have been higher if this potential selection bias did not exist. Finally, our retrospective cohort was too small to allow a meaningful analysis of delayed graft function, slow graft function, and technical failure. In conclusion, the length of the right renal vein on CTA correlates with ex vivo length after laparoscopic kidney procurement, but this does not hold true for the left renal vein. Our data indicate that there was no significant influence of right renal vein length on recipient outcome measures.

\section{Conflict of interest}

The authors state no conflict of interest. 


\section{References:}

1. Legendre C, Canaud G, Martinez F: Factors influencing long-term outcome after kidney transplantation. Transpl Int, 2014; 27(1): 19-27

2. Tsoulfas G, Agorastou P, Ko D et al: Laparoscopic living donor nephrectomy: is there a difference between using a left or a right kidney? Transplant Proc, 2012; 44(9): 2706-8

3. Bagul A, Frost JH, Mathuram Thiyagarajan $U$ et al: Extending anatomic barriers to right laparoscopic live donor nephrectomy. Urology, 2012; 79(2): 465-69

4. Buell JF, Edye M, Johnson $M$ et al: Are concerns over right laparoscopic donor nephrectomy unwarranted? Ann Surg, 2001; 233(5): 645-51

5. Diner EK, Radolinski B, Murdock JD, Ghasemian SR: Right laparoscopic donor nephrectomy: the Washington Hospital Center experience. Urology, 2006; 68(6): 1175-77

6. Özdemir-van Brunschot DM, Warlé MC, van der Jagt MF et al: Surgical team composition has a major impact on effectiveness and costs in laparoscopic donor nephrectomy. World J Urol, 2015; 33(5): 733-41

7. Yarlagadda SG, Coca SG, Garg AX et al: Marked variation in the definition and diagnosis of delayed graft function: a systematic review. Nephrol Dial Transplant, 2008; 23(9): 2995-3003
8. Nel D, Vogel J, Muller E et al: Slow early graft function: a neglected entity after renal transplantation. Nephron Clin Pract, 2012; 120(4): c200-4

9. Vilar E, Varagunam M, Yaqoob MM et al: Creatinine reduction ratio: a useful marker to identify medium and high-risk renal transplants. Transplantation, 2010; 89(1): 97-103

10. Jellison FC, Shah SK, Mashni JW Jr et al: Vessel length following laparoscopic donor nephrectomy: impact of vascular ligation technique on allograft vessel length. J Endourol, 2008; 22(5): 973-77

11. Gures N, Gurluler E, Berber I et al: Comparison of the right and left laparoscopic live donor nephrectomies: a clinical case load. Eur Rev Med Pharmacol Sci, 2013; 17(10): 1389-94

12. Hoda MR, Greco F, Wagner S et al: Prospective, nonrandomized comparison between right- and left-sided hand-assisted laparoscopic donor nephrectomy. Transplant Proc, 2011; 43(1): 353-56

13. Abrahams HM, Meng MV, Freise CE, Stoller ML: Pure laparoscopic right donor nephrectomy: step-by-step approach. J Endourol, 2004; 18(3): 221-25; discussion 225

14. Hellegering J, Visser J, Kloke $\mathrm{HJ}$ et al: Deleterious influence of prolonged warm ischemia in living donor kidney transplantation. Transplant Proc, 2012; 44(5): 1222-26 